\title{
Development of a redox polymer based on poly(2-hydroxyethyl methacrylate) for disposable amperometric sensors
}

\author{
Chiara Zanardi a,*, Laura Pigani a , Giulio Maccaferri ${ }^{\text {a,b }}$, Micaela Degli Esposti ${ }^{\text {c }}$, Paola Fabbri ${ }^{\text {d }}$, \\ Paolo Zannini ${ }^{\text {a }}$, Renato Seeber ${ }^{\text {a,b }}$ \\ a Department of Chemical and Geological Sciences, Università di Modena e Reggio Emilia, Via G. Campi 103, 41125 Modena, Italy \\ b Istituto di Sintesi organica e Fotoreattività (ISOF), Consiglio Nazionale della Ricerca (CNR), via Gobetti 101, 40129 Bologna, Italy \\ c Dipartimento di Ingegneria Enzo Ferrari, Università di Modena e Reggio Emilia, Strada Vignolese 905, 41125 Modena, Italy \\ d Dipartimento di Ingegneria Civile, Chimica, Ambientale e dei Materiali, Università di Bologna, via Terracini 28, 40131 Bologna, Italy
}

\section{A R T I C L E I N F O}

\section{Article history:}

Received 15 October 2015

Received in revised form 30 October 2015

Accepted 30 October 2015

Available online 26 November 2015

\section{Keywords:}

Electrode coatings

Electrocatalysis

Hydroxyethyl methacrylate

Ferrocenylmethyl methacrylate

Polymer membranes

Hydroquinone

\begin{abstract}
A B S T R A C T
Membranes based on poly(2-hydroxyethyl methacrylate), chemically modified with ferrocene functionalities, are proposed as novel coatings for the development of disposable amperometric sensors. They are massproduced on an inert support through an UV photo-induced polymerization, forming self-standing films that can be transferred on an electrode surface. Thanks to the characteristic of the functionalized methacrylate matrix to rapidly and reversibly swell by incorporation of large amounts of water, the ferrocene moieties are in intimate contact with the electrolytic solution. They can activate effective electrocatalytic processes that can be exploited in the field of amperometric sensing. The performance of the methacrylate coating with respect to the determination of hydroquinone benchmark analyte has been tested both in a static solution and in a flux cell.
\end{abstract}

(c) 2015 Elsevier B.V. All rights reserved.

\section{Introduction}

Amperometric sensors are acknowledged to be very efficient devices for food, environmental, clinical analyses and for process control. In the development of effective electrode systems, electrode coatings play the most meaningful role, imparting the device specific physico-chemical properties and corresponding analytical peculiarities. In particular, the acquisition of electrocatalytic activity is often sought, in order to detect target analytes at low potential values, where redox processes due to the solvent medium and to additional electroactive species present in the solution are absent [1]. To reach this goal, a suitable redox mediator is often fixed on the electrode [2]. In this context, a critical aspect is represented by the poor stability of the anchoring and by the time consuming procedures often required for the periodical renewal of the electrode functionality. An effective approach to overcome both these problems is represented by the use of disposable electrode coatings that covalently bond the redox mediator.

Poly(2-hydroxyethyl methacrylate) (pHEMA) forms a hydrogel in water. Self-standing films can be easily obtained through UVinduced photo-polymerization of 2-hydroxyethyl methacrylate (HEMA) in the presence of a photo-initiator. Molecules possessing

\footnotetext{
* Corresponding author.

E-mail address: chiara.zanardi@unimore.it (C. Zanardi).
}

a suitable functionality can be included by co-polymerization, stably linking the functional group to the pHEMA matrix. By following this approach, functionalized pHEMA membranes were successfully used for the development of an optical sensor for $\mathrm{pH}$ determination [3]. In that case, the sensing element, namely fluorescein, was linked to a methacrylate monomer in order to be stably bonded to pHEMA membrane; the sensor quickly responded to variable solution $\mathrm{pH}$, in a flow cell system.

pHEMA-based thin films have been also proposed as coatings for amperometric biosensors [4]: a solid film was directly obtained on the electrode surface by photo-polymerization of a drop of HEMA and vinylferrocene mixture. The resulting modified electrode was tested as an enzymatic biosensor for the determination of glucose. Furthermore, a different biocatalytic sensor was obtained by simply including ferrocene in the PHEMA material during UV-polymerization process [5].

It is however evident that the number of applications of pHEMA in the field of sensing, and in particular of amperometric sensing, is quite low.

In the present study, ferrocenylmethyl methacrylate (Fc-MMA) was co-polymerized with HEMA in order to build up a redox polymer in which the ferrocene/ferriciunium ion $\left(\mathrm{Fc} / \mathrm{Fc}^{+}\right)$redox couple is covalently bonded to the polymer chains. The resulting membranes consist of self-standing films that can be mass-produced by UV photo-induced polymerization on an inert support and easily transferred onto the 
electrode surface just before use. Thanks to the capability to swell once in contact with water, the Fc redox active residues inside the membrane are in intimate contact with the electrolytic solution: the $\mathrm{Fc} / \mathrm{Fc}^{+}$redox couple acts as the redox mediator with respect to proper substrates in solution. In the present article, as a proof of concept, the electrode system was tested as a sensor for hydroquinone (HQ) determination, both in static and in flux electrochemical cells.

\section{Material and methods}

\subsection{Synthesis of the pHEMA based membranes}

All reagents used for the synthesis of pHEMA-based membranes were from Sigma-Aldrich. Basically, two different starting solutions have been prepared. In a first one, $0.50 \mathrm{~g}(3.8 \mathrm{mmol})$ of HEMA, $0.01 \mathrm{~g}(3.3 \mu \mathrm{mol})$ of Fc-MMA (95\% pure), $0.30 \mathrm{~g}$ of water and $0.03 \mathrm{~g}(0.2 \mathrm{mmol})$ of photo-initiator, namely 2-hydroxy-2methylpropiophenone, were mixed in a sonicator for $20 \mathrm{~min}$. In a second one, $0.050 \mathrm{~g}$ ( $0.46 \mathrm{mmol})$ of hydrophilic sodium methacrylate and $0.01 \mathrm{~g}(5.0 \mu \mathrm{mol})$ of a bifunctional crosslinking agent, namely ethylene glycol dimethacrylate, were added to the former recipe, and the amount of water was increased to $0.50 \mathrm{~g}$. In both cases, a number of similar membranes, possessing $0.39 \mathrm{~mm}$ mean thickness (measured by a Borletti MDE1 digital micrometer) were simultaneously obtained: drops ( $6 \mu \mathrm{l}$ each) of pristine solution were deposited onto a polypropylene foil and exposed to UV light (Dymax Light-Curing Flood-Lamp System Model 5000, $4.9 \mathrm{~W} / \mathrm{cm}^{2}$ ) for a time suitable to achieve solidification. In particular, 180 and $240 \mathrm{~s}$ time lengths were adopted for the first and the second mixture, leading to $\mathrm{p}$ (HEMA-Fc)* (two-component copolymer) and p(HEMA-Fc) (fourcomponent copolymer), respectively. In order to evidence the effect induced by the presence of Fc moieties, membranes have been also synthesized with similar pristine solutions, in absence of Fc-MMA, obtaining pure $\mathrm{p}(\mathrm{HEMA})^{*}$ and $\mathrm{p}(\mathrm{HEMA})$, respectively.

\subsection{Analytical tests}

Dry $\mathrm{p}(\mathrm{HEMA})^{*}$ and $\mathrm{p}(\mathrm{HEMA}-\mathrm{Fc})^{*}$ membranes were positioned on the electrode surface and wet with a drop of water, inducing stable adhesion to electrode substrate.

$\mathrm{p}$ (HEMA) and $\mathrm{p}$ (HEMA-Fc) membranes were conditioned before use by immersion in water for at least $10 \mathrm{~min}$. In this step Fc-MMA not included in the co-polymer chain is released in solution, whereas the volume of the membranes increases of about ten times. The wet membranes were then placed on a Pt electrode and fixed by an O-ring and an external cap in order to achieve best fixing of the coating.

Modified electrodes were tested in a $0.1 \mathrm{M}$ phosphate buffer solution (PBS) at $\mathrm{pH}=7.0$. Known amounts of HQ were added, in order to define the performance of the sensing system. Cyclic voltammetry (CV) was the voltammetric technique chosen, sweeping the potential from -0.3 to $+0.6 \mathrm{~V}$ at $0.02 \mathrm{Vs}^{-1}$ potential scan rate.

Tests were also performed in a flux cell $(0.35 \mathrm{ml} / \mathrm{min}$ flux rate $)$ in a configuration typical of flow injection analysis: $100 \mu \mathrm{l}$ of a solution containing HQ at given concentrations was periodically added to the cell through a loop.

\subsection{Instrumentation}

All electrochemical measurements were performed with an Autolab PGSTAT12 (Ecochemie) potentiostat/galvanostat. A $2 \mathrm{~mm}$ diameter Pt disk (Metrohm, Herisau, Switzerland) was used as the working electrode; a GC rod was the auxiliary electrode and an aqueous $\mathrm{Ag} / \mathrm{AgCl}$, $3 \mathrm{M} \mathrm{KCl}$ (Metrohm) was the reference. The working electrode was polished subsequently with $0.05 \mu \mathrm{m}$ alumina powder, then rinsed with distilled water in an ultrasonic bath before use.
Thermogravimetric analyses on dry and wet membranes were performed with Netzsch STA 309 simultaneous TGA-DTA analyzer, in static air, on 19.1 and $5.0 \mathrm{mg}$ of sample, respectively, in a Pt crucible; $5{ }^{\circ} \mathrm{C} / \mathrm{min}$ heating rate till $800{ }^{\circ} \mathrm{C}$.

ICP-MS analyses were performed with a HR/MC/ICP-MS Neptune (Thermo Fisher Scientific) after complete mineralization of the membranes in an UltraClave microwave digestor (Milestone).

XRF analyses have been performed on the conditioned membrane, with a Bruker axs analyzer.

\section{Results and discussion}

$\mathrm{p}($ HEMA-Fc)* membranes have been firstly tested in PBS, leading to record the typical voltammogram reported in Fig. $1 \mathrm{~A}$. The increasing height of the anodic-cathodic peak system in subsequent potential cycles, ascribed to the $\mathrm{Fc} / \mathrm{Fc}^{+}$moieties bonded to the polymer chains $[4,6-10]$, indicates the progressive swelling of the membrane due to the inclusion of counterions from the solution to compensate the charges brought by the $\mathrm{Fc}^{+}$residues formed. Water molecules also
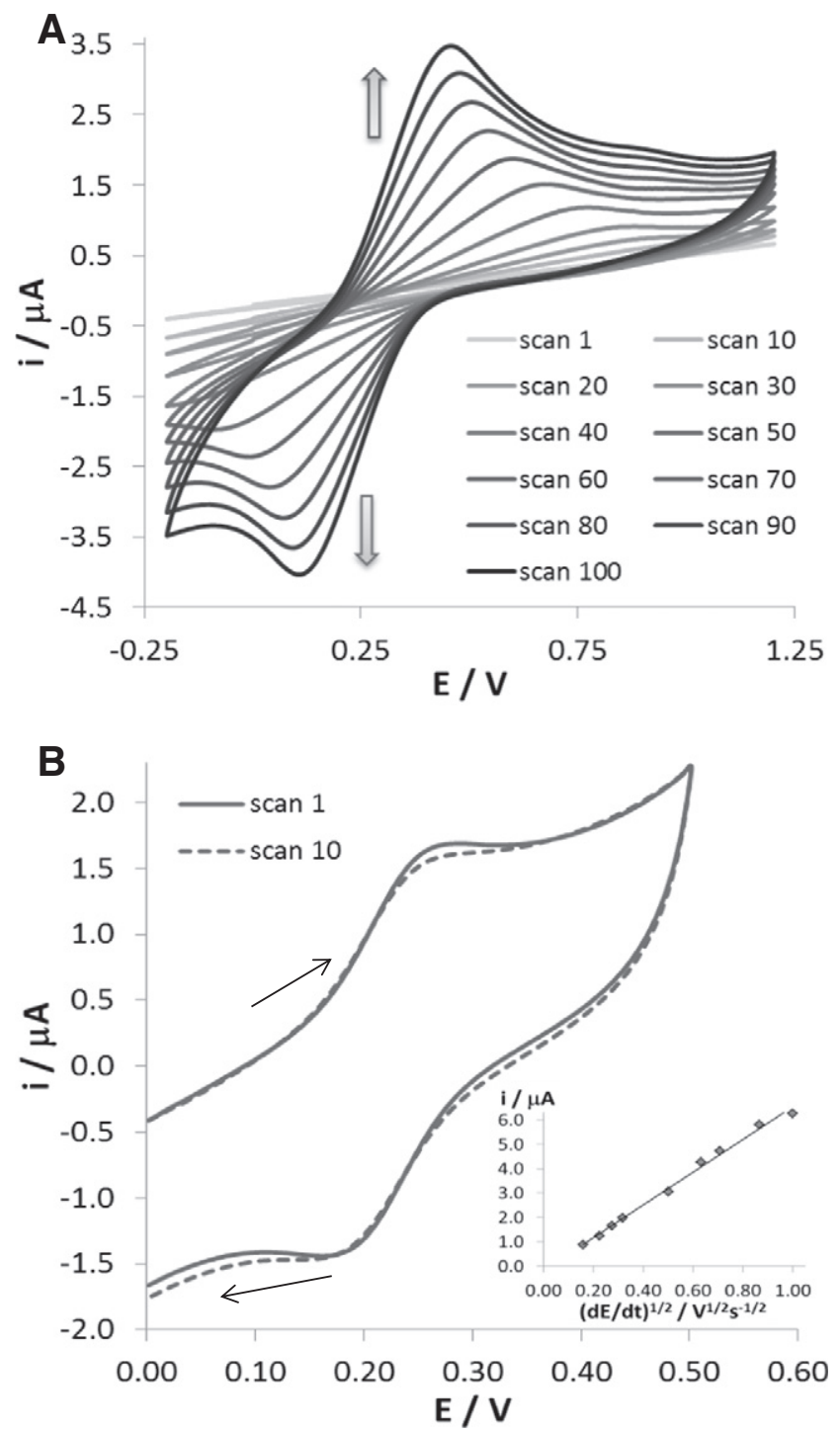

Fig. 1. Different CV scans of $A) p(H E M A-F c)^{*}$ and $\left.B\right) p(H E M A-F c)$ modified Pt electrodes in PBS; $\mathrm{v}=0.10$ and $0.05 \mathrm{Vs}^{-1}$, respectively. Grey arrows in $\mathrm{A}$ indicate the evolution of the voltammogram scan by scan. Inset in B reports the plot of current peak values vs. square root of the potential scan rate. 
contribute to polymer swelling. Such a trend indicates the porous nature of the methacrylate hydrogel membrane, which possesses floppy channels suitable to allow solvated ions and solvent entrance.

However, when dipped in solutions containing easily oxidizable species, such as $\mathrm{H}_{2} \mathrm{O}_{2}$, ascorbic acid or $\mathrm{HQ}$, no change in the voltammetric picture, ascribable to electrocatalytic action of the coating, is detectable. This suggests that $\mathrm{Fc}^{+}$sites cannot approach the cited substrate to act as the oxidant. Actually, by using simple $\mathrm{p}(\mathrm{HEMA})^{*}$ coatings we could verify that the membrane is not permeable to similar redox-active species: no signal ascribable to analyte reaching the underlying electrode surface is recorded.

The literature dealing with applications of pHEMA evidences that the dimensions of the hydrogel pores can be increased by addition of a suitable amount of hydrophilic sodium methacrylate in the polymerization solution [11]. However, we could verify that too much water can enter the membranes in this case, causing loss of their physical stability. Then, a cross-linking agent, namely ethylene glycol dimethacrylate monomer, was added to the reaction mixture to form a covalently bonded 3D network. The formulation of the polymerization mixture finally reported in the experimental section, indicated as p(HEMA-Fc), satisfies the request of a material that is stable under hydrated form and is also permeable to analytes from the solution.

The voltammetric behavior of $\mathrm{p}$ (HEMA-Fc) in PBS is reported in Fig. 1B. As observed, the material shows, since the very first scan, an anodic-cathodic peak system due to the $\mathrm{Fc} / \mathrm{Fc}^{+}$residues. The peak current is proportional to the square root of the potential scan rate in the range $0.02-1.00 \mathrm{Vs}^{-1}$ (see inset of Fig. 1B), indicating that the number of redox active sites is constant in the sweep rate interval explored [12].

The amount of Fc-MMA monomer units resulting actually included in the polymer matrix was determined by ICP analysis, revealing that it is as low as $0.33 \%$. In other words, only one unit over about 300 structural units is redox active. Such a low amount of Fc units in the fourcomponent film is justified by the poor solubility of Fc-MMA in the synthesis mixture that limits the amount of redox mediator finally linked to the polymer film. For this reason, measurements at varying the concentration of Fc-MMA in the starting solution have not been possible. Despite the low molar fraction of Fc moieties in the membrane, the current peak observed is surprisingly high, indicating that quite a large number of electroactive sites are in contact with the solution. As predicted, in fact, the membrane shows a very high tendency to incorporate water molecules: the volume increases of about ten times after dipping it for about $10 \mathrm{~min}$ in water. TGA analysis performed on dry and wet membranes indicates that the amount of water included is around $80 \% w / w$; membranes containing such a large percentage are stable only thanks to the presence of bifunctional acrylate units. The thickness of the wet membrane finally results of about $0.93 \mathrm{~mm}$.

Since a certain amount of Fc-MMA is lost during the conditioning process in water, the reproducibility of the concentration of Fe finally present inside each $\mathrm{p}$ (HEMA-Fc) membrane was tested by XRF analyses on four wet membranes, by recording the signal due to Fe absorption in four different points of each membrane. ANOVA analysis indicates that, at a probability level of 5\% for type I errors [13], the fraction of structural units containing Fc residues in the four membranes is not significantly different.

The electrocatalytic activity of Fc sites in the coating has been tested with respect to HQ oxidation. When the Pt electrode is coated with a $\mathrm{p}$ (HEMA) membrane, a peak system relative to an anodic oxidation affected by significant overvoltage is recorded. It resembles quite closely the response obtained on bare Pt surfaces [14] (Fig. 2A). This indicates that pores of the membrane are suitable to allow percolation of such electroactive species down to the electrode surface. When using $\mathrm{p}$ (HEMA-Fc) membranes, the presence of Fc residues induces a significant lowering of the potential at which HQ oxidation occurs, as expected for a redox mediator fixed on the electrode. As observed in the inset of Fig. 2B, the peak height is proportional to HQ concentration and is well reproducible on four different electrode coatings (mean RSD $=10.6 \%$ ).
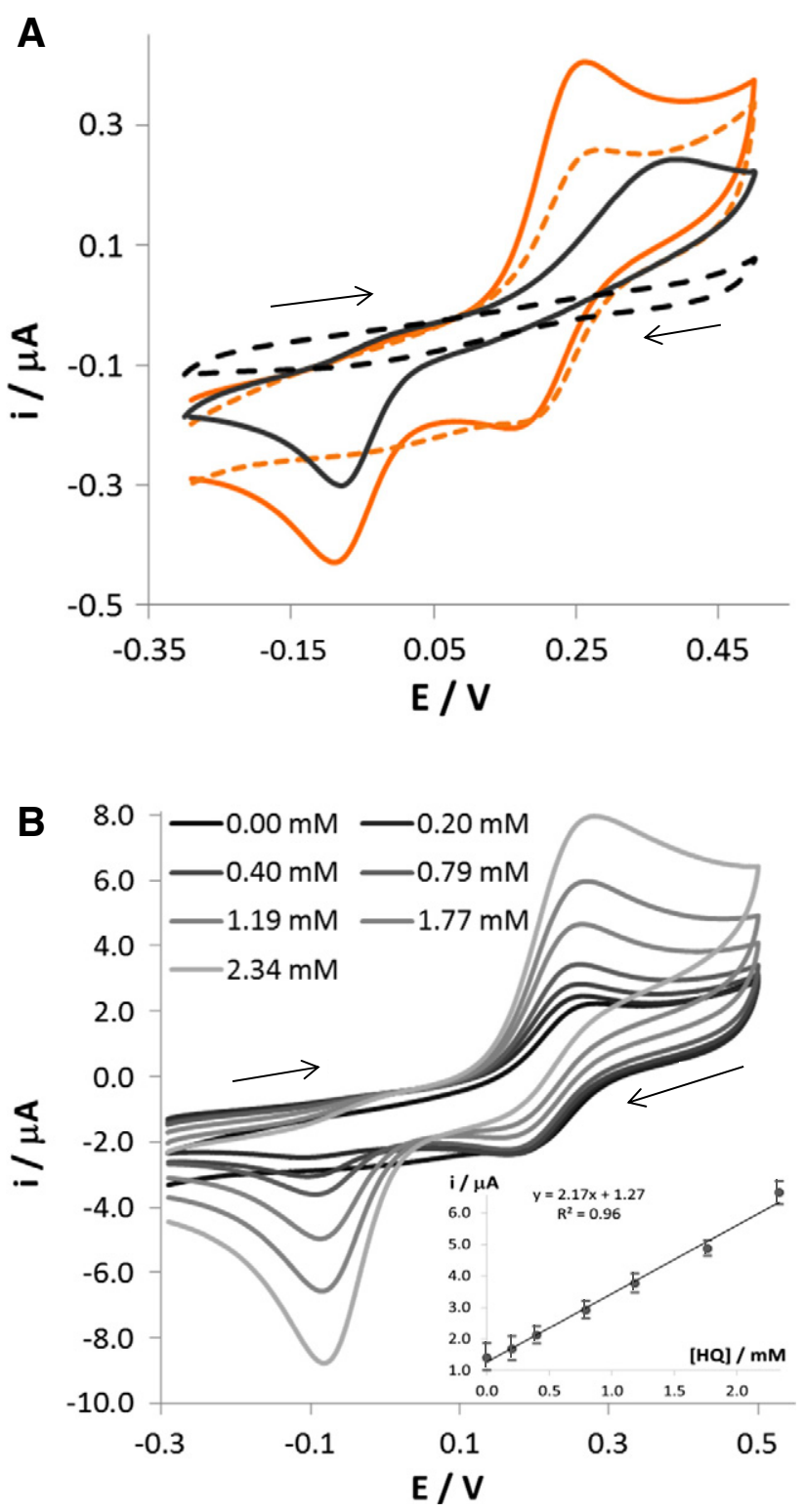

Fig. 2. Steady state voltammetric curves recorded on A) p(HEMA) (black lines) and $\mathrm{p}$ (HEMA-Fc) (orange lines) modified Pt electrodes in the absence (dotted lines) and in the presence (solid lines) of $1 \mathrm{mM} \mathrm{HQ}$; B) p(HEMA-Fc) modified Pt electrode at different concentrations of $\mathrm{HQ}$; $0.1 \mathrm{M}$ PBS $(\mathrm{pH} 7.0), \mathrm{v}=0.02 \mathrm{Vs}^{-1}$. Inset in $\mathrm{B}$ reports the calibration plots calculated by considering the results obtained by 4 membranes; the error bar length accounts for two-times the standard deviation.

In order to test the applicability of the sensor for rapid analytical determinations, as those most often requested by on-line (intermitted) detection [15], flow injection analyses have been performed. As observed in Fig. 3, four replicates of the analysis on the same HQ solution result in quite repeatable signals ( $R D S=1.8 \%$ ). Furthermore, calibration curves registered by random additions method, in the 0.04-1.50 mM concentration range, show quite a good linearity $\left(\mathrm{R}^{2}=0.99\right)$, demonstrating the high stability of the coating and the lack of memory effects in so critical conditions. Although specific tests aimed at defining the stability of the membrane with respect to the number of measurements have not been made, we could verify that such a linearity range is preserved by performing as many as 40 subsequent injections of analyte solution into the electrochemical cell. 


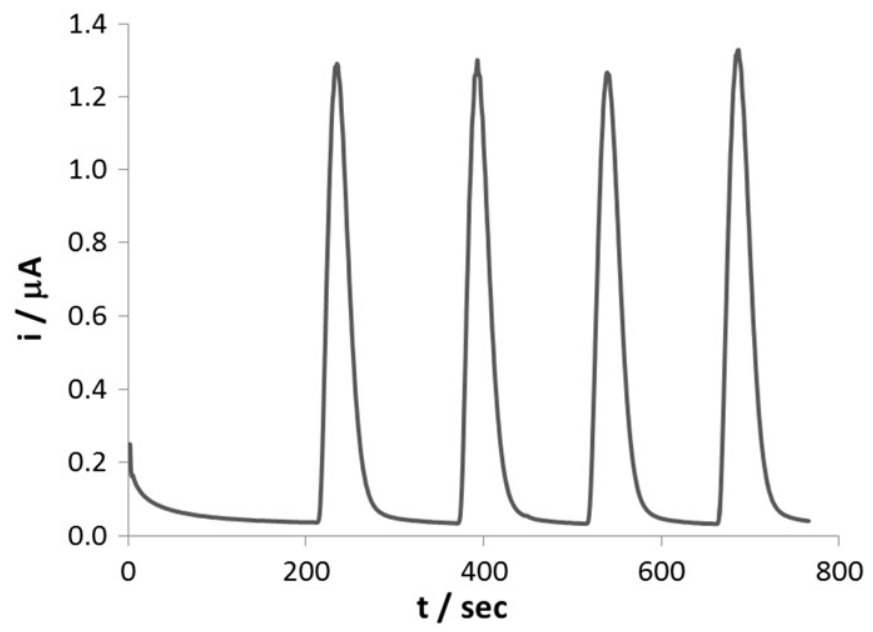

Fig. 3. Amperometric detection performed at $\mathrm{E}=+0.35 \mathrm{~V}$ with $\mathrm{p}$ (HEMA-Fc) modified electrode in a flux cell $(0.35 \mathrm{ml} / \mathrm{min}$ flux rate $)$ in $0.1 \mathrm{M}$ PBS in the absence and in the presence of $0.5 \mathrm{mM} \mathrm{HQ}$; the signal increment corresponds to the periodical injection of $100 \mu \mathrm{l}$ of analyte solution.

\section{Conclusions}

A stable polymeric membrane consisting of a four-component methacrylate polymer is developed and proved to be effective as a redox mediator in anodic oxidation. Quantitative tests have been carried out with respect to HQ solutions. Interestingly, the sensor system exhibits quite fast responses, resulting effective in static solutions as well in flow injection analyses over a wide substrate concentration range. Also considering the excellent repeatability of the responses, the system should be carefully considered for a number of analytical applications even including the monitoring of flowing systems. Furthermore, the good reproducibility of membranes allows us to propose the device as a disposable one, which can be very rapidly mass-produced.

The proof of concept of this novel polymeric electrode modifier definitely suggests us to explore similar systems further on, substituting the $\mathrm{Fc} / \mathrm{Fc}^{+}$redox couple with different mediators and even considering the possible inclusion of enzymes for the development of biocatalytic electrode systems.

\section{Acknowledgments}

The authors kindly acknowledge the University of Modena and Reggio Emilia for the financial support in the framework of the strategic line "Nano- and emerging materials and systems for sustainable technologies".

\section{References}

[1] R. Seeber, L. Pigani, F. Terzi, C. Zanardi, Amperometric sensing. A melting pot for material, electrochemical, and analytical sciences, Electrochim. Acta 179 (2015) 350-363.

[2] R. Seeber, F. Terzi, C. Zanardi, Functional Materials in Amperometric Sensing. Polymeric, Inorganic, and Nanocomposite Materials for Modified ElectrodesBook series: in: F. Scholz (Ed.), Monographs in Electrochemistry, Springer, New York, ISBN: 9783-662-45102-1, 2014.

[3] L. Ferrari, L. Rovati, P. Fabbri, F. Pilati, Disposable fluorescence optical pH sensor for near neutral solutions, Sensors 13 (2013) 484-499.

[4] L. Sim Bean, L. Yook Heng, B.M. Yamin, M. Ahmad, Photocurable ferrocenecontaining poly(2-hydroxyl ethyl methacrylate) films for mediated amperometric glucose biosensor, Thin Solid Films 477 (2005) 104-110.

[5] R. Mohammad, M. Ahmad, L.Y. Heng, An amperometric biosensor utilizing a ferrocene-mediated horseradish peroxidase reaction for the determination of capsaicin (chili hotness), Sensors 13 (2013) 10014-10026.

[6] G. Inselt, L. Szabo, The effect of the nature and of the concentration of counter ions on the electrochemistry of poly(vinylferrocene) polymer film electrodes, Electrochim. Acta 31 (1986) 1381-1387.

[7] P. Audebert, P. Calas, G. Cerveau, R.J.P. Corriu, N. Costa, Modified electrodes from organic-inorganic hybrid gels containing ferrocene units covalently bonded inside a silica network, J. Electroanal. Chem. 372 (1994) 275-277.

[8] H. Ju, D. Leech, Electrochemistry of poly(vinylferrocene) formed by direct electrochemical reduction at a glassy carbon electrode, J. Chem. Soc. Faraday Trans. 93 (1997) 1371-1375.

[9] J. Wang, M.M. Collinson, Electrochemical characterization of inorganic organic hybrid films prepared from ferrocene modified silanes, J. Electroanal. Chem. 455 (1998) 127-137.

[10] F. Darsun, S.K. Ozoner, A. Demirci, M. Gorur, F. Yilmaz, E. Erhan, Vinylferrocene copolymer based biosensors for phenol derivatives, J. Chem. Technol. Biotechnol. 87 (2012) 95-104.

[11] Y.A. Han, E.M. Lee, B.C. Ji, The physical properties of poly(2-hydroxyethyl methacrylate) copolymer hydrogels used as intravaginal rings, Chin. J. Polym. Sci. 27 (2009) 359-366.

[12] R.W. Murray, Chemically modified electrodes, in: A.J. Bard (Ed.)Electroanalytical Chemistry, vol. 13, Marcel Dekker, New York 1984, pp. 191-368.

[13] D.L. Massart, B.G.M. Vandeginste, S.N. Deming, Y. Michotte, L. Kaufman, Chemometrics: A TextbookBook series: in: B.G.M. Vandeginste, L. Kaufman (Eds.), Data Handling in Science and Technology, Elsevier, Amsterdam, 1988.

[14] S. Lupu, F. Parenti, L. Pigani, R. Seeber, C. Zanardi, Differential pulse techniques on modified conventional-size and microelectrodes. Electroactivity of poly[4,4bis(butylsulfanyl)-2,2-bithiophene] coating towards dopamine and ascorbic acid oxidation, Electroanalysis 15 (2003) 715-725.

[15] J. Callis, D.L. Illman, B.R. Kowalski, Process analytical, Anal. Chem. 59 (1987) 624A-637A 